\title{
Procedimiento metodológico para el estudio de procesos servuctivos de restauración
}

\author{
Methodological procedures for the study of restoration process servuctivos
}

\author{
Cecilia Parra Ferié ${ }^{1 *}$, Bertha Soraya Rhea González², \\ Conrado Ernesto Gómez Román ${ }^{3}$ \\ ${ }^{1}$ Escuela Superior Politécnica Agropecuaria de Manabí, Ecuador \\ ${ }^{2}$ Universidad Técnica del Norte, Ecuador \\ ${ }^{3}$ Metropolitana de Santiago, Chile
}

Recibido el 30 de enero del 2017; aceptado el 22 de mayo del 2018

Disponible en Internet el 20 de noviembre de 2018

\begin{abstract}
Resumen
En la presente investigación se abordan cuestiones básicas relacionadas con la gestión de los servicios y dentro de ésta el papel rector de la servucción como proceso de creación del servicio. Se realiza el diseño de un procedimiento que permite realizar el despliegue de las diferentes etapas que conducen a la instrumentación del concepto del nuevo servicio y se presentan las bases metodológicas para su implementación. Se aplican herramientas de consenso como el método Delphi mediante el trabajo con expertos para la determinación de los atributos del servicio de restauración que son valorados por los clientes. También se demuestra la factibilidad de emplear la Matriz Importancia-Presencia Percibida para establecer las prioridades de mejora en aquellos atributos que sean más importantes para los clientes y que estén menos adecuados
\end{abstract}

Códigos JEL: M1, M31, M32

Palabras clave: Servicios restauranteros; Procesos servuctivos; Atributos del servicio.

*Autor para correspondencia.

Correo electrónico: parraferiececilia@gmail.com (C. Parra Ferié)

La revisión por pares es responsabilidad de la Universidad Nacional Autónoma de México. 


\begin{abstract}
In this research addresses basic issues related to the management of services and within it the leading role of the servuction as service creation process. It is performed the design of a procedure that allows the deployment of the different stages leading to the implementation of the new service concept and methodological basis for implementation. Are applied tools like Delphi consensus method applied by working with experts to determine the attributes of service options that are valued by customers. The feasibility of using Matrix Importance - Perceived Presence to set priorities for improvement in those attributes that are most important to customers and are less suitable is also demonstrated
\end{abstract}

JEL Codes: M1, M31, M32

Keywords: Restaurateurs services; Servuctivos processes; Service attributes

\title{
Introducción
}

En la década de los 90 del pasado siglo, el número de empleos en organizaciones de servicio en los países industrializados como Estados Unidos, Francia, Canadá, Japón y Gran Bretaña aumentó del 60 al 80\%. Varias publicaciones muestran como ha existido un cambio de la economía orientada hacia los servicios, revelando que por ejemplo en 1984 el sector de los servicios representa el $66 \%$ de la economía de los Estados Unidos y un $58 \%$ de la de los países de la Comunidad Económica Europea. En España, en 1987, el sector de los servicios representaba el 61,5 \% del Producto Interno Bruto (PIB); en 1993 en los países desarrollados los servicios representaban más del $60 \%$ del PIB, siendo el sector que generaba mayor volumen de empleo (Grönroos, 1994).

Ya en este siglo XXI, los servicios alcanzan en algunos países el $80 \%$ del PIB. En este sentido Aboal, Crespi y Rubalcaba (2015) enfatizan en que la productividad del sector servicios es cada vez más importante para promover el crecimiento y la equidad tanto en las economías desarrolladas (OCDE, 2010; Comisión Europea, 2009) como, y particularmente, en aquellos en desarrollo, a los que cabe mencionar Cuba, Ecuador, Venezuela, Brasil, entre otros.

Autores como Aboal et.al (2015, pág 10) plantean que "los servicios son fundamentales en los procesos de innovación de los otros sectores de la economía. Esto se opone a la visión tradicional en la que los sectores de servicios se consideran poco innovadores y poco generadores de innovación. De hecho, el sector servicios ha sido visto tradicionalmente como menos innovador que el sector manufacturero y jugando solo un papel marginal en el proceso de innovación. Como resultado de esto, las políticas nacionales de innovación han prestado poca atención a los servicios, y las empresas del sector servicios han sido descuidadas en los programas de innovación patrocinados por los gobiernos".

Estos autores refieren además que el rápido crecimiento de los servicios en la economía mundial es innegable. Sin embargo, las razones por las que los servicios han adquirido tal prominencia son diferentes en cada país. Por tanto, hay al menos ocho razones que explican el crecimiento de los servicios:

1. Tendencias de la productividad en sectores específicos (enfermedad de costos de Baumol)

2. El crecimiento del ingreso 
3. La acumulación de capital humano

4. Los cambios tecnológicos y las tecnologías de la información y la comunicación (TIC).

5. El cambio organizacional

6. La integración de los servicios

7. La globalización del comercio

8. El Estado, las regulaciones y el cambio institucional y social

Dado el ámbito en que se desarrolla la investigación, es necesario realizar un análisis de las particularidades de los servicios desde su concepción, es decir, desde la óptica servuctiva.

En una empresa de servicios hay tantas servucciones como servicios. En algunas empresas de servicios hay servucciones "tradicionales" con la intervención del personal y servucciones "automatizadas" realizadas por el cliente sin tener contacto con el personal. Todas estas servucciones tienen un elemento común y, en general, único: el cliente (Parra Ferié, 2009).

Cuando se habla de producción, se piensa en una fábrica y en la fabricación de bienes tangibles. Pero, ¿es fabricado un servicio del mismo modo que un bien cualquiera? El concepto de servucción aporta una visión particular de la gestión de las empresas, que se contempla como el sistema de producción del servicio, es decir, la parte visible de la organización en la que se producen, distribuye y consumen los servicios. En esta investigación se pretende dar este enfoque servuctivo.

El término "Servucción" define el proceso de creación de servicio (Eigler y Langeard, 1989). Estos profesores franceses conceptualizan la Servucción como "la producción del servicio". Es la organización sistemática y coherente de todos los elementos físicos y humanos de la relación cliente-empresa necesaria para la realización de una prestación de servicio cuyas características comerciales y niveles de calidad han sido determinadas.

La servucción es todo el engranaje detrás de la prestación de un buen servicio. Es por ello importante el estudio de los sistemas de servicios y buscar nuevas formas de gestionar sus procesos servuctivos.

¿Qué se necesita para fabricar un servicio? En pocas palabras, podría decirse que se necesita mano de obra, un soporte físico y un beneficiario, es decir, un cliente (Eigler y Langeard, 1989). Haciendo una representación simplificada de un sistema de servucción, se presentan los siguientes elementos:

- El cliente. El consumidor está implicado en la fabricación del servicio. Es un elemento primordial y su presencia es indispensable.

- El soporte físico. Se trata del soporte material necesario para la producción del servicio. Contempla dos categorías: los instrumentos necesarios para el servicio (muebles, maquinarias) y el entorno material en el que se desarrolla el servicio (locación, edificio, decorado).

- El personal de contacto. Son las personas empleadas por la empresa que están en contacto directo con los clientes.

- El servicio. Es el resultado de la interacción de los tres elementos de base que son el cliente, el soporte físico y el personal de contacto. Este resultado constituye un beneficio que debe satisfacer la necesidad del cliente. La gran diferencia entre la fabricación de un servicio y la fabricación de un producto es que el cliente es un integrante fundamental del sistema de servucción: es a la vez productor y consumidor. Por lo tanto, es clave entender 
la secuencia de los actos de participación en la servucción que el cliente lleva a cabo para beneficiarse del servicio ofrecido.

Bajo el paradigma de este enfoque, juega un papel preponderante la evaluación desde la perspectiva del cliente, para lo cual Duque (2005) plantea que la calidad percibida y su evaluación depende de las características específicas del servicio, de los clientes y del contexto en el cual se desenvuelven, luego se debe tener en cuenta el concepto de servicio a evaluar y su caracterización. Esto implica que, como afirman los representantes de las escuelas europea y americana de la calidad Grönroos y Parasuramant, la calidad percibida de los servicios es la mejor forma de conceptualizar la calidad en el ámbito de los servicios (Parra Ferié, 2009). Stefano, Casarotto Filho \& Godoy (2010) agregan que cabe a los prestadores de servicio conocer las expectativas de sus clientes para buscar mejorías de desempeño que favorezcan una percepción siempre buena, buscando hacer investigaciones junto a sus clientes, a fin de conocer y obtener mejoría continúa, aumentando de esta forma, la calidad percibida por estos.

En este sentido Briceño y García (2008) plantean que en el siglo XXI, el cliente es cada vez más exigente en cuanto al cumplimiento de criterios mínimos de calidad de servicio y derechos como usuario debido a que recibe más información y porque los componentes de la competitividad de las empresas (adecuados estándares de calidad, cantidad, costos, y tiempo en la prestación de los ser vicios) aumentan la oferta en el mercado de los servicios, lo que hace necesario cuidar al máximo el logro de la satisfacción del cliente, como fortaleza en la gestión de la calidad del servicio.

Otro aspecto importante a tener en cuenta en la gestión de los servicios, es la identificación y medición de los elementos tangibles e intangibles, mediante el uso de diferentes modelos de medición de la calidad del servicio. Morillo Moreno et. al. (2011) abordan cuestiones aún válidas definidas por autores como Denton (1991) y Pride y Ferrel (1997), los cuales plantean la necesidad de realizar la medición de la calidad del servicio para que los proveedores de estos conozcan como sus clientes los evalúan.

Los elementos conceptuales abordados hasta el momento tienen particular connotación en los procesos de servicios de restauración, siendo necesario realizar una mirada a los mismos.

La restauración como vocablo puede conceptualizarse en diferentes contextos como el artístico, político, siendo el más común su aplicación en los procesos que se llevan a cabo para preservar o devolver la originalidad de un bien cultural. Sin embargo, en la presente investigación se hace referencia a la restauración como actividad económica, es decir la que se desarrolla en un local denominado "restaurante".

La actividad de restauración se puede resumir como la conversión de los factores de producción (alimentos, capital, trabajo) mediante la producción y el servicio, en productos solicitados por un cliente que busca el consumo, la satisfacción y el bienestar (Cañizal, 1996 citado por Cisneros Mustelier y Hechavarría Viera, 2011).

En consecuencia (Gómez, 2006) plantea que debe verse como un sistema que se compone de tres elementos fundamentales:

1. Los bienes materiales. Están constituidos por los alimentos en sí, lo constituyen tanto el elemento principal de cada plato como el acompañante. Su calidad depende de la materia prima empleada y del conjunto de procesos que tienen lugar en la actividad de restauración, y que contribuyen a la calidad de la oferta. 
2. Los atributos del producto. Están determinados por los elementos que acompañan a los alimentos, los cuales pueden ser físicos (mobiliario, cubertería, vajilla) y de servicio (amabilidad del empleado, profesionalidad).

3. La extensión del producto. Es el conjunto de satisfacciones que presenta el cliente como resultado de la calidad de la oferta y el servicio recibido. Depende del trato que se recibe, del menú, ambientación del salón. Es un elemento que permite valorar de manera general como se siente el cliente en el restaurante. La restauración tiene entre sus objetivos básicos satisfacer los gustos y preferencia de los clientes y dar placer; es un servicio de obligado consumo, por lo que no se puede dejar a un lado el desarrollo del mismo, si se tiene en cuenta que el cliente del siglo XXl es cada día más exigente y sus necesidades y expectativas son cada vez mayores.

A lo anterior se suma el hecho de que la actividad de restauración es compleja, más aún si se desea brindar un servicio eficiente y a la vez que origine ganancias, por lo que se hace necesario una gestión con objetivos claramente definidos y la importancia de contar con personal calificado, comprometido con la labor que realiza (Cisneros Mustelier y Hechavarría Veira, 2011).

Hay que tener en cuenta que en la actividad de la Restauración convergen dos elementos importantes: la transformación de productos para la elaboración de la oferta y la prestación del servicio, los cuales constituyen procesos claves ya que son percibidos por el cliente y son la razón de ser de la organización (Espinosa, 2010).

Autores como Betancourt Ramírez; Aldana de Vega y Gómez Betancourt (2014) abordan tres elementos importantes para el estudio comparativo del desempeño de restaurantes familiares y no familiares, los cuales tienen puntos de coincidencia con la investigación realizada, como son:

- Calidad: Abarca la presentación, manipulación y estado de la comida. Allí se tiene en cuenta la presentación del plato y las bebidas, la temperatura, el sabor, los alimentos y para algunos platos el término o punto de cocción y originalidad en la presentación.

- Servicio: Hace referencia al desempeño del personal, la capacidad para asesorar al cliente, el conocimiento de los platos de la carta, la amabilidad, la agilidad y la puntualidad.

- Ambiente: Contempla elementos como decoración, temática, ambientación musical, iluminación, comodidad de las sillas, limpieza del establecimiento, vajilla, recepción y fachada; esto de acuerdo con la especialidad del restaurante.

Los fundamentos teóricos revisados, permiten plantear la pertinencia del diseño de un procedimiento donde es posible el despliegue de las diferentes etapas que conducen a la instrumentación del concepto del nuevo servicio, lo cual será tratado a continuación.

\section{Materiales y métodos}

Para el desarrollo de la investigación se emplearon métodos científicos como induccióndeducción, análisis-síntesis y analógico; a través de los cuales se logró recopilar la información necesaria para llegar a la propuesta de un procedimiento metodológico para el diseño de procesos servuctivos teniendo en cuenta el concepto del nuevo servicio y su aplicación en el ámbito de los servicios de restauración.

Entre las principales técnicas aplicadas se destacan el método Delphi para la obtención de 
los atributos que caracterizan el proceso de servicio de restauración, Triángulo de Füller en la comparación por pares, la Matriz Importancia-Presencia Percibida.

En la concepción del procedimiento se tuvo en cuenta que todos los recursos y las energías de la empresa están dedicados a la realización de un concepto de servicio que generalmente no está formalizado, ya que evoluciona con el tiempo. Por lo tanto, hay que desarrollar la fórmula del nuevo servicio.

\section{Resultados}

Para llegar al planteamiento del procedimiento metodológico que más adelante se presenta, se tuvo en cuenta la fórmula del nuevo servicio, la cual comprende cinco decisiones claves que se interesan por el concepto, el segmento, la servucción, la oferta y la imagen (Hernández González, 2008).

\section{Un concepto de servicio único}

Las empresas de servicio que quieren convertirse en líderes no pueden contentarse con tener actividades: deben apoyarse en conceptos fuertes. Para conseguir esta fuerza, han de contar con una serie de factores:

- Creatividad y movilización alrededor del concepto: La creatividad es indispensable y desemboca en un concepto de servicio que es un conjunto coherente de actividades existentes, de funciones conocidas, de distintas culturas, es decir, elementos que hasta ahora estaban separados. La movilización de las energías de la empresa es tanto una necesidad como una consecuencia, y facilita en la empresa la comunicación de las misiones, de la función y del estilo de cada uno.

- Concepto y la diferenciación: Hoy en día se imita fácilmente una actividad, pero difícilmente un concepto. La búsqueda del concepto de servicio se basa, por lo tanto, en concretar la diferenciación y comunicarla a la clientela. De este modo se obtendrá un concepto fuerte.

- Concepto único: comprende la originalidad y la unicidad. El concepto de servicio saca su fuerza de su originalidad. Para ser originales, no se necesita "revolucionar" el mercado. Esto significa que también se puede ser originales (y que esta originalidad sea duradera) tomando un conjunto de elementos que por separado sean conocidos y con poco impacto, pero que juntos tienen un gran poder de anticipación. Por otro lado, la unicidad es la que confiere la fuerza de penetración en el mercado y, para ello, el concepto ha de ser único. Una buena gestión consistirá en elegir el mejor concepto, que será aquel que se acople mejor al segmento de mercado que se considere más interesante.

\section{Un segmento de mercado único e identificable}

- El enfoque de mercado segmentado: Un concepto de servicio es una respuesta coherente a cierto tipo de necesidades. Es necesario entonces formular e implementar una estrategia de segmentación, que garantice la satisfacción de las necesidades de un mercado más específico y menos abarcador.

- Las variables de comportamiento y los atributos del servicio: Para segmentar el mercado deben tenerse en cuenta las variables de comportamiento para medir el perfil de los que tienen la misma necesidad, así como la situación en la que se encuentra el consumidor. 
- Tamaño del segmento y fuerza del concepto: El tamaño de los segmentos varía mucho. Un concepto fuerte es aquel que conlleva la adhesión de un gran número de consumidores que están en los bordes del segmento y que, modificando sus expectativas y comportamiento, integran el mismo.

\section{Una servucción especializada y fácil de desmultiplicar}

Hay que prever en detalle el funcionamiento de la servucción y de sus tres grandes categorías de componentes (personal en contacto, soporte físico y cliente) con la preocupación de conservar la simplicidad. Esto es necesario por dos razones: se favorece la rapidez de desarrollo de los procesos, que es una ventaja competitiva que lleva al éxito, y además se permite a los clientes aprender rápidamente lo que se espera de ellos.

También hay que evitar la complejidad de la servucción que es un peligro mortal para todos los grandes sistemas de servicio.

\section{Una oferta limitada fácil de estructurar}

Debe tratarse de una oferta estructurada que responda a una doble condición: por un lado, hacer resaltar la prestación que es percibida por el consumidor; y por otra parte, limitar el número de servicios periféricos para dejar a la servucción toda su sencillez y, por tanto, su fiabilidad. Este es el campo de decisión más difícil de abordar.

\section{Una imagen de servicio clara y fácil de comunicar}

Ya que los servicios son intangibles, es una tarea difícil crear una comunicación eficaz. Aunque utilicemos de forma inteligente los medios de comunicación no será suficiente, ya que la imagen del servicio también depende, y en gran medida, de la interacción de la empresa con sus clientes.

Estos clientes, a su vez, difunden la imagen cuando comparten su experiencia con el entorno y, si la imagen que perciben es positiva, beneficiará a la empresa en gran medida. Ésta es una razón por la que la imagen debe ser muy clara. La imagen además es una decisión clave que depende fuertemente de los otros cuatro campos de decisión. Si el concepto no es único y simple, la imagen no será clara y significativa.

\section{Procedimiento para el diseño de procesos servuctivos}

En la figura 1 se muestra el procedimiento con las etapas o pasos a cumplimentar para realizar el diseño de un proceso servuctivo teniendo presente el concepto del nuevo servicio 


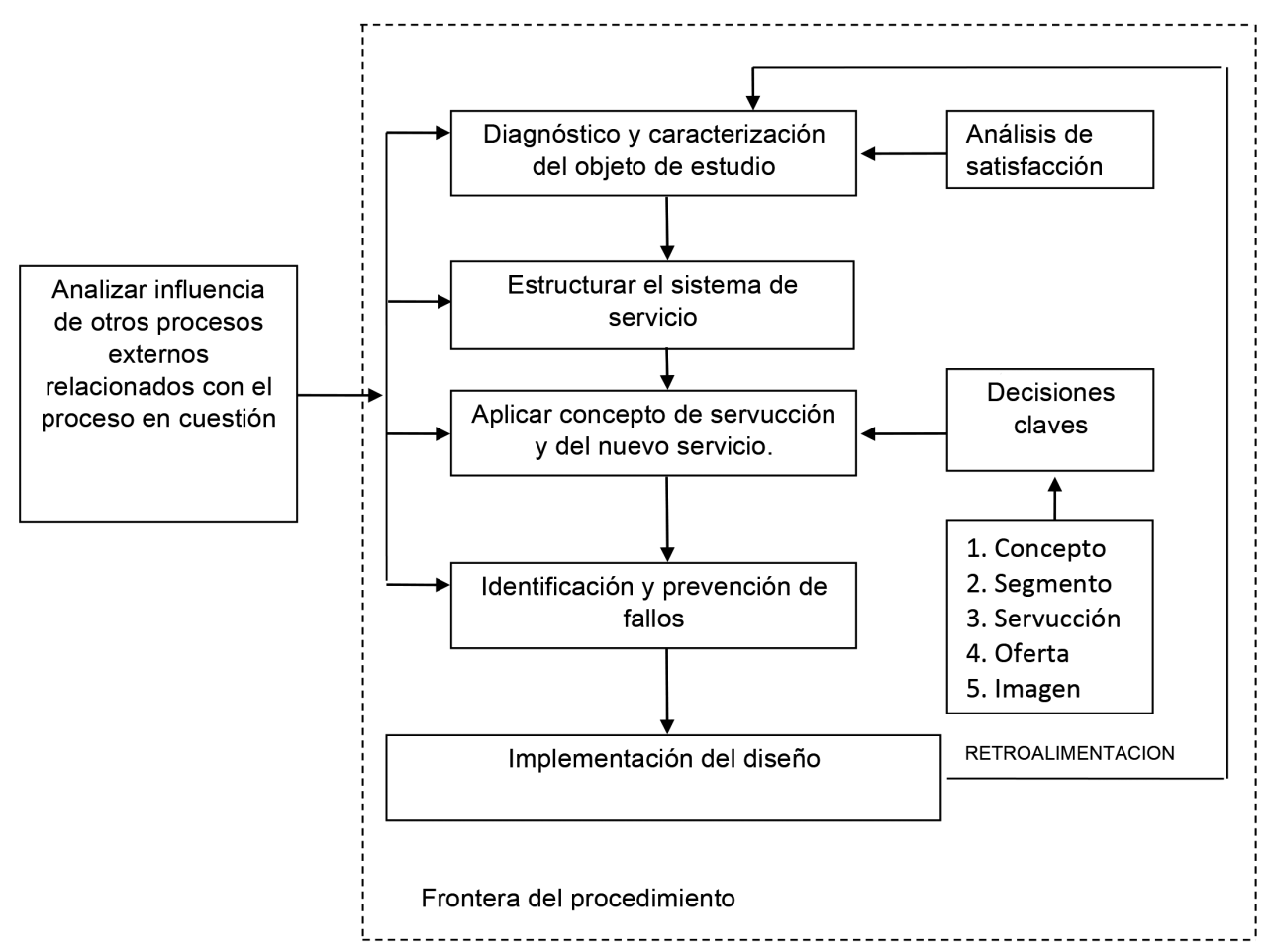

Figura 1: Procedimiento propuesto para el diseño del proceso servuctivo. Fuente: Elaboración propia.

La primera etapa del procedimiento es el diagnóstico y caracterización del objeto de estudio. Según (Hernández Torres, 2008), el diagnóstico debe ser aprovechado para actuar por adelantado sobre los procesos, antes de que ocurran las desviaciones, indicando los posibles obstáculos que se opondrán al rumbo estratégico fijado, creando las condiciones para que los resultados coincidan con los objetivos, conforme a la característica del control de gestión moderno, referida a que éste debe orientarse más al futuro como control de gestión prospectivo.

En este procedimiento el diagnóstico consiste en realizar una caracterización del objeto de estudio, en la cual se vaya de lo general a lo particular. Realizar un análisis de la satisfacción del cliente con el proceso. Las posibles técnicas a aplicar en esta primera etapa son: Técnicas de análisis y recopilación de información, Encuestas para medir satisfacción del cliente con el proceso, Método de los expertos, Análisis Causa-Efecto, Flujograma.

La segunda etapa del procedimiento es la estructuración del sistema de servicio. El conjunto de servicios elementales, base y periféricos son entidades relacionadas entre sí que forman un sistema. Los elementos del sistema están formados por cada servicio elemental y su servucción y todos estos ellos tienen un elemento común: el cliente. En esta etapa se debe estructurar el sistema de forma tal que todos los procesos y subprocesos apunten hacia el cliente y su satisfacción. Las posibles técnicas a aplicar son: la técnica encuesta, flujogramas y otras. 
La tercera etapa del procedimiento consiste en aplicar el concepto de Servucción y del nuevo servicio. Definir la idea exacta de la servucción a realizar.

Los procesos servuctivos son todos aquellos donde se realiza o brinda un servicio por la organización, con la participación del cliente como beneficiario del servicio y representado a través de su propiedad, que interactúa con el personal de contacto sobre la base o utilización de un soporte físico (Parra Ferié, 2009).

La propuesta es en base a relacionar las cinco decisiones claves que se deben tomar antes de aplicar el concepto de nuevo servicio:

- Concepto, el cual comprender características claves e imprescindibles en su uso que deben ser la creatividad, la originalidad, y la diferenciación.

- Segmento, donde se debe abarcar solo un perfil no todo el mercado para poder ser más objetivos en nuestra forma de proceder a la hora de brindar un servicio.

- Servucción, la cual debe ser especializada y fácil de desmultiplicar dentro de ella entra el personal en contacto, el soporte físico y el cliente.

- La oferta, limitada y fácil de estructurar, para no cometer errores y conformar una estructura que no sea la más adecuada a la demanda.

- La imagen, uno de los factores decisivos en la creación de un servicio es la comunicación, si la imagen no es clara y fácil de comunicar no despierta interés y lo que no interesa no se compra. Estas decisiones claves se deben tener en cuenta en todo el proceso y en cada una de las acciones que se ejecuten durante el mismo.

Las posibles técnicas a aplicar en esta etapa son: Técnicas de análisis y recopilación de información, segmentación del mercado, benchmarking, diagrama de servucción, entre otras.

La cuarta etapa del procedimiento es la identificación y prevención de fallos, tras el análisis de cuáles son las desviaciones. Se toma acción en la etapa de ejecución del servicio, para prevenir que los errores se conviertan en defectos. Si no es posible prevenir el riesgo, entonces al menos se debe detectar. (Parra Ferié, 2009).

El control de fallos tiene un impacto directo en los resultados del proceso, el seguimiento dado a los posibles fallos permitirá al responsable de cada subproceso tomar decisiones. Dentro de las cuales no debe obviar las cinco decisiones claves. La prevención de los fallos, constituye en sí misma una oportunidad de mejora del proceso. Es por ello, que se propone aplicar un modelo de recopilación de información según (Parra Ferié, 2005) que viabiliza la identificación de las Oportunidades de Mejora.

El análisis de causas constituye la base de una toma de decisiones oportunas y efectivas. Se debe controlar la ejecución de los proyectos de acción seleccionados, evaluando los resultados a nivel local, evaluando la efectividad del proceso de mejoramiento sobre el cumplimiento de los objetivos inicialmente planteados a la entidad, en el horizonte de tiempo considerado.

La quinta y última etapa del procedimiento es la implementación del diseño. Como señala (Amozarrain, 1999) la fase de implantación puede prolongarse en el tiempo, por lo que es necesario desarrollar un plan concreto con la definición de responsables y plazos para cada uno de los hitos.

En esta etapa el enfoque de proceso es fundamental. Autores como Harrington (1998), Medina León y Nogueira Rivera (2012) hacen énfasis en la necesidad de definir los procesos internos de la organización, como vía de alineación de los resultados proyectados con los objetivos organizacionales. 
Antes de implantar el nuevo proceso es necesario reflexionar acerca de las posibles resistencias al cambio y las posibles contramedidas a adoptar, de entre las que se pueden citar las siguientes:

- Comunicar y hacer partícipes a las personas que se verán implicadas en la puesta en práctica del nuevo proceso.

- Dar la formación y adiestramiento necesarios.

- Escoger el momento adecuado.

- Desarrollar una implantación progresiva: se procura iniciar esta con las personas más receptivas y con las de más prestigio entre sus compañeros.

En la representación esquemática del procedimiento anteriormente expuesto en la figura 1, se puede apreciar la línea de retroalimentación que parte de la implementación hasta el diagnóstico.

Analizar la influencia de otros procesos externos que influyen en el buen funcionamiento del proceso es un aspecto del procedimiento de importancia ineludible, ya que estos se interrelacionan en un todo e integran el sistema, donde la comunicación juega un papel primordial.

Aplicación parcial del procedimiento en el estudio del proceso de Alimentos y Bebidas del Hotel Playa Caleta de Varadero.

A manera de validación práctica del procedimiento antes expuesto a continuación se muestra la aplicación parcial del mismo en el Hotel Playa Caleta de Varadero, llegando a determinarse el Nivel de Servicio ofertado a partir de una serie de análisis realizados con un equipo de expertos previamente seleccionados para este fin. Se aplicaron un conjunto de técnicas y herramientas que permitieron arribar a conclusiones importantes acerca de la calidad del servicio ofertado en el proceso de Alimentos y Bebidas.

Para la determinación de los atributos del servicio de restauración por el equipo de expertos en el tema de gestión de los servicios y de manera más particular en los de restauración, se aplicó el Método Delphi, cuyas características propiciaron su instrumentación en la investigación.

Este método se desarrolla a través de rondas, y como ventaja adicional se tiene que no es necesario hacer coincidir a los expertos en el mismo tiempo y lugar, siendo posible utilizar expertos ubicados en diferentes lugares geográficos.

Los expertos a los cuales se hace alusión en esta etapa son especialistas seleccionados los cuales conforman el grupo de trabajo.

Autores como Nogueira Rivera (2002); Negrín Sosa (2003); Parra Ferié (2009) y Diéguez Matellán (2008) reconocen la necesidad de avalar el grado de conocimiento y confiabilidad de los expertos a partir de la aplicación del cuestionario de Competencia del Experto (Oñate Ramos (2002, el cual es un instrumento de gran utilidad, particularmente cuando se requiere recopilar información de la experiencia y conocimiento de un grupo de personas relacionadas con el objeto de la investigación.

Por cuestiones prácticas, teniendo en cuenta además que este instrumento parte de la valoración del propio experto se consideró en su selección tener en cuenta las variables que se mencionan a continuación:

1. Experiencia en la comercialización o prestación de los servicios de alimentos y bebidas: esta variable es fundamental, pues atribuye una fuerte fiabilidad a los argumentos expuestos por cada experto.

2. Capacitación profesional actual: la idea que mientras más conocimiento y grado de 
profesionalidad dentro de una empresa de servicios tengan mayor va hacer el nivel de servicio ofertado considerándola como una variable dominante para el proceso.

Para la selección de los expertos se utiliza el coeficiente de competencia K, el cual se determina de acuerdo con la opinión del experto sobre su nivel de conocimiento con respecto al problema que se está resolviendo (Kc) y con las fuentes que le permiten comprobar su valoración (Ka). El coeficiente de competencia se calcula de la siguiente forma:

$\mathrm{K}=(\mathrm{Kc}+\mathrm{Ka}) / 2$.

Donde:

Kc: Es el coeficiente de conocimiento del experto

Ka: Es el coeficiente de argumentación del experto

En el proceso de selección de los expertos, el coeficiente de competencia debe cumplir la condición de $\mathrm{K} \geq 0.7$, obteniéndose los resultados que se muestran en la Tabla 1 , después de procesar la información recopilada sobre los candidatos a integrar el grupo.

Tabla 1

Coeficientes de Competencia de los Expertos.

\begin{tabular}{lccccccc}
\hline Expertos & E1 & E2 & E3 & E4 & E5 & E6 & E7 \\
\hline $\begin{array}{l}\text { Coeficiente de } \\
\text { Competencia }\end{array}$ & 0.73 & 0.85 & 0.70 & 0.87 & 0.92 & 0.81 & 0.89 \\
\hline
\end{tabular}

Fuente: Elaboración propia

Como puede apreciarse, los candidatos muestran un coeficiente de competencia adecuado, por lo que el equipo de expertos quedó formado por 7 especialistas en el área de la restauración gastronómica.

\section{Determinación de las variables o atributos del servicio de restauración}

Para el estudio del proceso de servicio de restauración es necesario definir las variables o parámetros que lo caracterizan y que son valorados por los clientes durante su consumo.

Entre los diversos métodos para tomar decisiones no estructuradas sobre un sistema de variables para caracterizar un atributo tan intangible como los beneficios buscados por los clientes en el proceso de alimentos y bebidas, el método Delphi se considera útil y pertinente basado en las razones siguientes:

- El número de factores que es considerado por un grupo es mayor que el que podría ser tenido en cuenta por una sola persona. Cada experto podrá aportar a la discusión general la idea que tiene sobre el tema debatido desde su área de conocimiento.

- Imposibilidad de reunir a todos los expertos en el mismo lugar y en el mismo instante.

- La condicional profesional de evitar criterios unipersonales o subjetividad individual que pudieran restar credibilidad y fiabilidad a la investigación.

En la conformación del análisis mediante el método Delphi en la primera ronda se les 
presentó a los expertos un listado inicial de 24 variables, con el objetivo de que decidieran si bastaban para definir los beneficios buscados por los consumidores, o si es necesario adicionar o modificar alguno. Estas fueron:

1. Visibilidad de la instalación desde fuera

2. Cualidades físicas de las instalaciones

3. Singularidad de las instalaciones

4. Disponibilidad de aparcamiento

5. Proximidad al transporte público

6. Vistas del paisaje

7. Facilidades de acceso

8. Imagen proyectada

9. Comunicación y publicidad del producto

10. Variedad, tipo de cocina/especialidad

11. Tipo de servicio

12. Ambientación/Atmósfera del lugar

13. Climatización

14. Horario de servicio

15. Requerimientos de entrada

16. Relación calidad/precio

17. Facilidades de pago

18. Calidad del servicio

19. Habilidades mostradas por el personal

20. Visibilidad de la elaboración

21. Comida para llevar o servicio a domicilio

22. Cualidades físicas de las instalaciones o negocios cercanos

23. Singularidad de los negocios circundantes

24. Complementariedad de los negocios adyacentes

En la segunda ronda se procede a listar y presentar a los expertos las variables resultantes de la ronda anterior, con vista a que realicen la votación según los procedimientos establecidos; o sea, marcar con " 1 " aquellos elementos con los que estén de acuerdo y con "0" aquellos con los que no.

En este paso se calcula el coeficiente de concordancia a cada una de las variables en estudio, mediante la siguiente expresión:

$\mathrm{Cc}=(1-\mathrm{Vn} / \mathrm{Vt}) * 100$

Donde:

Cc: Coeficiente de concordancia expresado en tanto por ciento

Vn: Cantidad de votos negativos

Vt: Cantidad total de votos

El método Delphi establece que se seleccionan aquellas variables que cumplan como condición que su coeficiente de concordancia sea mayor que 0.7 
A continuación, se muestra en la Tabla 2 el análisis realizado por los expertos:

Tabla 2.

Votaciones realizadas por los expertos.

\begin{tabular}{|c|c|c|c|c|c|c|c|c|c|c|}
\hline Variables & E1 & E2 & $\begin{array}{l}\mathrm{E} \\
3\end{array}$ & $\begin{array}{l}\mathrm{E} \\
4\end{array}$ & $\begin{array}{l}\mathrm{E} \\
5\end{array}$ & $\begin{array}{l}E \\
6\end{array}$ & $\begin{array}{l}\mathrm{E} \\
7\end{array}$ & $\begin{array}{c}\text { Votos } \\
\text { negativos } \\
\text { por } \\
\text { variable }\end{array}$ & $\begin{array}{l}\text { Concordancia } \\
\text { por variable }\end{array}$ & Selección \\
\hline $\begin{array}{l}\text { 1. Visibilidad de la } \\
\text { instalación desde fuera }\end{array}$ & 1 & 0 & 1 & 0 & 1 & 0 & 1 & 3 & 0.571 & Eliminado \\
\hline $\begin{array}{l}\text { 2. Cualidades físicas de las } \\
\text { instalaciones }\end{array}$ & 1 & 1 & 1 & 1 & 0 & 1 & 1 & 1 & 0.857 & Seleccionado \\
\hline 3. Vistas del paisaje & 0 & 0 & 1 & 1 & 0 & 0 & 1 & 4 & 0.428 & Eliminado \\
\hline 4. Facilidades de acceso & 0 & 0 & 1 & 0 & 1 & 0 & 1 & 4 & 0.428 & Eliminado \\
\hline $\begin{array}{l}\text { 5. Comunicación y } \\
\text { publicidad del producto }\end{array}$ & 1 & 1 & 1 & 1 & 0 & 1 & 1 & 1 & 0.857 & Seleccionado \\
\hline $\begin{array}{l}\text { 6. Variedad, tipo de } \\
\text { cocina/especialidad }\end{array}$ & 1 & 1 & 1 & 1 & 0 & 1 & 1 & 1 & 0.857 & Seleccionado \\
\hline 7. Tipo de servicio & 1 & 1 & 1 & 1 & 1 & 1 & 1 & 0 & 1 & Seleccionado \\
\hline $\begin{array}{l}\text { 8. Ambientación/Atmósfera } \\
\text { del lugar }\end{array}$ & 1 & 1 & 1 & 0 & 0 & 1 & 1 & 2 & 0.711 & Seleccionado \\
\hline 9. Climatización & 1 & 0 & 1 & 1 & 1 & 1 & 1 & 1 & 0.857 & Seleccionado \\
\hline 10. Horario de servicio & 1 & 0 & 1 & 0 & 1 & 1 & 1 & 2 & 0.711 & Seleccionado \\
\hline 11. Requerimientos de entrada & 0 & 0 & 1 & 0 & 0 & 0 & 0 & 6 & 0.142 & Eliminado \\
\hline 12. Relación calidad/precio & 1 & 1 & 1 & 1 & 1 & 1 & 1 & 0 & 1 & Seleccionado \\
\hline 13. Calidad del servicio & 1 & 1 & 1 & 1 & 1 & 1 & 1 & 0 & 1 & Seleccionado \\
\hline $\begin{array}{l}\text { 14. Habilidades demostradas } \\
\text { por el personal }\end{array}$ & 1 & 1 & 1 & 1 & 1 & 1 & 1 & 0 & 1 & Seleccionado \\
\hline $\begin{array}{l}\text { 15. Visibilidad de la } \\
\text { elaboración }\end{array}$ & 0 & 0 & 0 & 0 & 1 & 1 & 1 & 4 & 0.428 & Eliminado \\
\hline $\begin{array}{l}\text { 16. Complementariedad de los } \\
\text { servicios de vino }\end{array}$ & 1 & 1 & 1 & 0 & 1 & 0 & 1 & 2 & 0.711 & Seleccionado \\
\hline
\end{tabular}

Fuente: Elaboración propia

Las variables o atributos seleccionados y que caracterizan el servicio de restauración son:

1. Cualidades físicas de la instalación

2. Comunicación y visibilidad del producto

3. Variedad, tipo de cocina/especialidad

4. Tipo de servicio

5. Ambientación/atmósfera del lugar

6. Climatización

7. Horario de servicio

8. Relación calidad-precio 


\section{Calidad del servicio}

10. Habilidades mostradas por el personal

11. Complementariedad de los servicios de vinos

Una vez definidos los atributos, se procedió a aplicar la encuesta a los clientes donde deben ponderar el nivel de importancia que le dan a cada atributo y por otro lado el nivel de presencia percibida, es decir, en qué medida perciben la presencia de este atributo en el servicio de restauración que consumen.

La encuesta se diseñó a partir de una escala tipo Likert de "1" a " 5 " puntos que servirán para evaluar tanto la Importancia ( $1=$ Nada importante, $2=$ Poco Importante, $3=$ Medianamente importante, 4= Importante, y 5= Muy importante) como el Grado de presencia percibida (1= Nada adecuado, 2= Poco adecuado, 3= Medianamente adecuado, 4= Adecuado, y 5= Muy adecuado), ya que como refieren Diéguez Matellán (2009), Pérez Gosende (2009) y Carreras Iler (2011) es una de las más utilizadas en las investigaciones comerciales y se considera puede abarcar todos los criterios existentes.

En la Figura 2 se presenta el instrumento aplicado, al cual se le realizó la comprobación de la fiabilidad y validez a partir de la medición de los coeficientes Alpha de Cronbach (a) y Coeficiente de Regresión Múltiple $\left(\mathrm{R}^{2}\right)$ respectivamente.

\begin{tabular}{|c|c|c|c|c|c|c|c|c|c|c|}
\hline \multirow{3}{*}{ Beneficios buscados } & \multicolumn{5}{|c|}{$\begin{array}{l}\text { Valore la importancia que usted le } \\
\text { concede a cada uno de los } \\
\text { siguientes elementos }\end{array}$} & \multicolumn{5}{|c|}{$\begin{array}{l}\text { ¿Cómo usted percibe la presencia } \\
\text { de estos elementos en los } \\
\text { servicios de alimentos y bebidas } \\
\text { que ofrece el Hotel Playa Caleta? }\end{array}$} \\
\hline & \multicolumn{2}{|c|}{ Nada Importsnte } & \multicolumn{3}{|c|}{$\rightarrow$ Muy Importsnte } & \multicolumn{5}{|c|}{ Nads sdecuado $\rightarrow$ Muy adecuado } \\
\hline & 1 & 2 & 3 & 4 & 5 & 1 & 2 & 3 & 4 & 5 \\
\hline $\begin{array}{l}\text { 1. Cualldades fislcas de las } \\
\text { Instalaclones }\end{array}$ & 口 & ㅁ & ㅁ & 口 & 口 & ㅁ & 口 & 口 & ㅁ & ㅁ \\
\hline $\begin{array}{l}\text { 2. Comunicacion y publicidad } \\
\text { del producto }\end{array}$ & ㅁ & ㅁ & $\square$ & $\square$ & 口 & $\square$ & $\square$ & 口 & $\square$ & ㅁ \\
\hline $\begin{array}{l}\text { 3. Varledad, tipo de } \\
\text { coclna/erpeclalldad }\end{array}$ & 口 & ㅁ & ㅁ & 口 & 口 & ㅁ & $\square$ & 口 & ㅁ & 口 \\
\hline 4. Tlpo de servicio & ㅁ & ㅁ & $\square$ & ㅁ & 口 & ㅁ & $\square$ & ㅁ & $\square$ & ㅁ \\
\hline $\begin{array}{l}\text { 5. Amblentacion/Atmosfera } \\
\text { del lugar }\end{array}$ & ㅁ & 口 & ㅁ & 口 & 口 & ㅁ & $\square$ & 口 & ㅁ & ㅁ \\
\hline 6. Cilmatizacion & 口 & 口 & $\square$ & 口 & 口 & ㅁ & 口 & ㅁ & ㅁ & ㅁ \\
\hline 7. Horarlo de serviclo & 口 & 口 & $\square$ & 口 & 口 & $\square$ & 口 & 口 & $\square$ & 口 \\
\hline 8. Relacion calldad/preclo & ㅁ & ㅁ & 口 & 口 & 口 & ㅁ & 口 & 口 & ㅁ & ㅁ \\
\hline 9. Calldad del serviclo & ㅁ & 口 & $\square$ & $\square$ & 口 & $\square$ & $\square$ & 口 & $\square$ & $\square$ \\
\hline $\begin{array}{l}\text { 10. Habillidades mostradas } \\
\text { por el personal }\end{array}$ & ㅁ & ㅁ & ㅁ & 口 & 口 & ㅁ & 口 & 口 & 口 & ㅁ \\
\hline $\begin{array}{l}\text { 11. Complementariedad de } \\
\text { los servicios de vino }\end{array}$ & 口 & ㅁ & ㅁ & 口 & ㅁ & ㅁ & $\square$ & ㅁ & ㅁ & ㅁ \\
\hline
\end{tabular}

Figura 2. Instrumento aplicado para la determinación del grado de importancia y de presencia percibida de los atributos del servicio de restauración. Fuente: Elaboración propia.

Los valores obtenidos con el uso del paquete estadístico SPSS versión 20.0 fueron Alfa de Cronbach igual a 0.941 y $\mathrm{R}^{2}$ igual a 0.919 , siendo ambos valores superior a 0.70 , demostrando que el instrumento es fiable y válido, tomando como referencia los criterios de George y Mallery (2003, p. 231). 
Esta encuesta se complementa con la Matriz Importancia-Presencia Percibida de los atributos del servicio de restauración estudiado.

En la figura 3 se muestra dicha matriz:

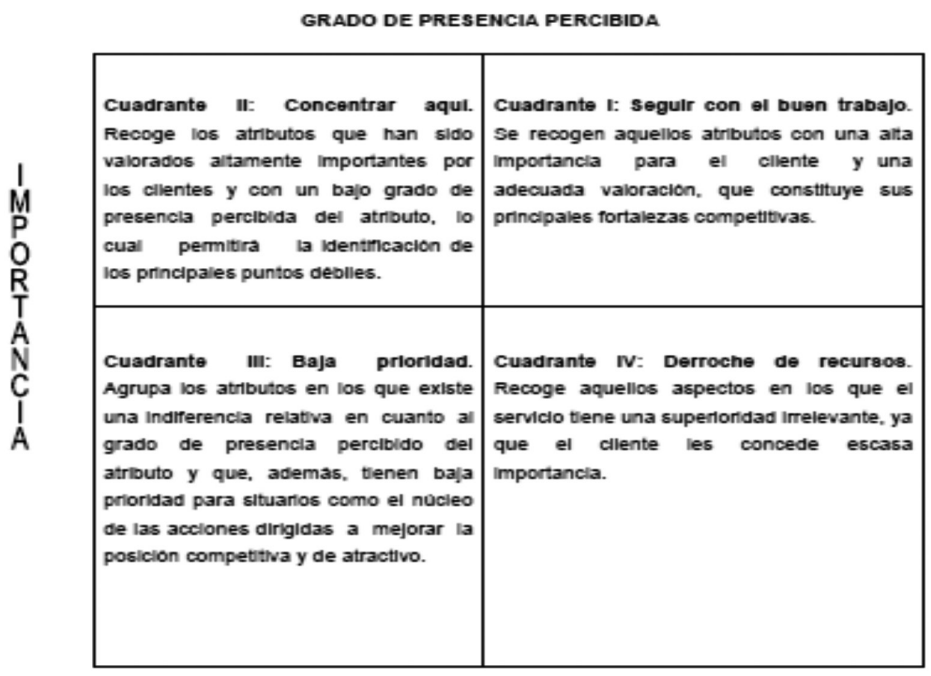

Figura 3. Matriz Importancia-Presencia Percibida

Esta matriz tiene como antecedente la propuesta por Mantilla y James (1977), para estudiar la imagen de destinos turísticos. Shetna (1982), determinó que cuanto mayor es la diferencia entre la importancia concedida a un atributo y adecuación en un producto determinado, mayor es la insatisfacción del consumidor hacia el producto, y por tanto la necesidad de realizar una acción correctora.

Es un procedimiento que muestra la importancia relativa de varios atributos y su grado de presencia percibida. De esta manera, la utilización de un análisis de este tipo puede ser de gran ayuda para los responsables de la gestión turística, ya que facilita la identificación de aspectos o atributos a los cuales se les debe dedicar una mayor atención, asignándoles más recursos y programas para mejorar.

La representación gráfica se realiza en un eje de coordenadas, cuyo origen es el valor medio obtenido para todos los atributos en cuanto a su importancia y grado de presencia percibida del atributo, aunque también puede asignarse al grado de presencia percibida del atributo un nivel deseado. Los datos se presentan en mapas, en los que cada atributo es ubicado en función del valor concedido a su importancia y grado de presencia percibida del atributo. La representación gráfica de los datos exige que cada atributo se ubique en una de las cuatro celdas, facilitando una rápida y visual evaluación de los atributos de atractivos del objeto de estudio.

Los resultados de la encuesta aplicada a una muestra de clientes del Hotel objeto de estudio, se ubican en la Matriz Importancia-Presencia Percibida, pudiendo establecerse las prioridades para la mejora en aquellos atributos que sean más importantes para los clientes y que estén menos adecuados, tal como se muestra en la figura 4. 
Las acciones de mejora para aumentar la presencia percibida de estos beneficios en el proceso deben centrarse en los beneficios que se ubican en los cuadrantes Alta importancia - Alto grado de presencia percibida (Puntos fuertes del proceso) y Alta importancia - Bajo grado de presencia percibida (Puntos críticos), así como en la brecha existente en cada uno de ellos.

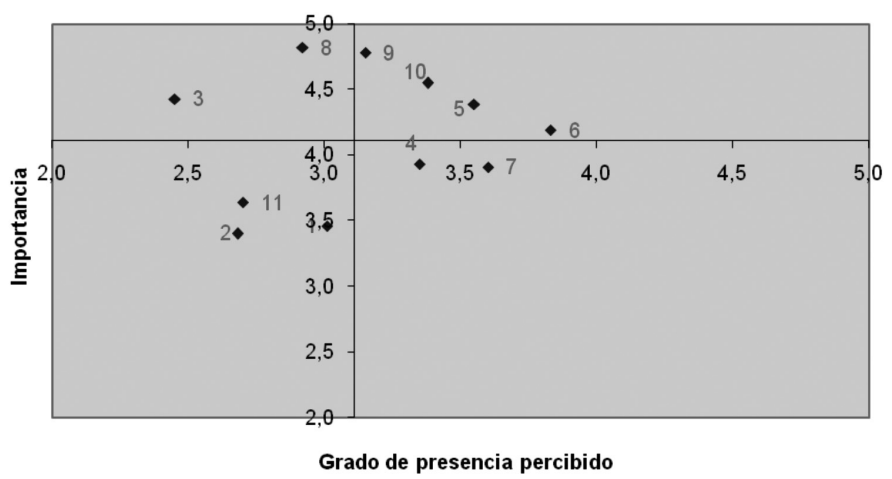

Figura 4. Ubicación de los valores resultantes de la encuesta aplicada a los clientes en la Matriz Importancia-Presencia percibida. Fuente: Elaboración propia.

Leyenda:

1. Cualidades físicas de las instalaciones

2. Comunicación y publicidad del producto

3. Variedad, tipo de cocina/especialidad

4. Tipo de servicio

5. Ambientación/Atmósfera del lugar

6. Climatización

7. Horario de servicio

8. Relación calidad/precio

9. Calidad del servicio

10. Habilidades demostradas por el personal

11. Complementariedad de los servicios de vino

De igual manera, se pudo determinar las brechas existentes entre lo que perciben los clientes (estado actual) y lo que desearían (estado deseado), lo cual se muestra en la figura 5. 


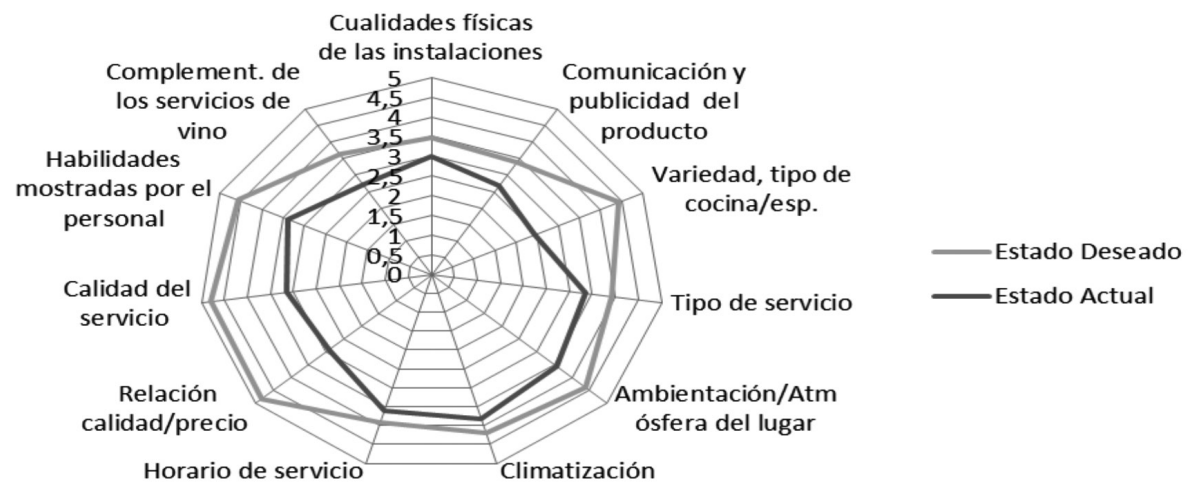

Figura 5. Comparación del estado actual con el estado deseado.

Fuente: Elaboración propia

Para los clientes encuestados del Hotel Playa Caleta, el diagrama anterior muestra que el total de los beneficios buscados considerados en el estudio reciben puntuaciones del grado de presencia percibida por debajo de su importancia, o sea, para este grupo de consumidores el servicio ofrecido por la organización en Alimentos y Bebidas no logró satisfacer ninguno de los beneficios buscados por ellos.

Los beneficios que tienen una brecha negativa entre lo que desea el cliente y lo que actualmente éste percibe aparecen en la tabla 3. En todos los casos se observa una brecha negativa entre ambos estados, lo cual indica que los clientes se encuentran insatisfechos con cada uno de los beneficios buscados.

Tabla 3.

Diferencias entre el estado actual y deseado de los clientes

\begin{tabular}{lccc}
\hline Beneficios buscados & Estado deseado & Estado actual & Brecha \\
\hline 1-Cualidades físicas de las instalaciones & 3.46 & 3.01 & -0.45 \\
2-Comunicación y publicidad del producto & 3.4 & 2.68 & -0.72 \\
3-Variedad, tipo de cocina/especialidad & 4.43 & 2.45 & -1.98 \\
4-Tipo de servicio & 3.93 & 3.35 & -0.58 \\
5-Ambientación/Atmósfera del lugar & 4.39 & 3.55 & -0.84 \\
6-Climatización & 4.19 & 3.83 & -0.36 \\
7-Horario de servicio & 3.91 & 3.6 & -0.31 \\
8-Relación calidad/precio & 4.82 & 2.92 & -1.9 \\
9-Calidad del servicio & 4.78 & 3.15 & -1.63 \\
10-Habilidades mostradas por el personal & 4.55 & 3.38 & -1.17 \\
11-Complementariedad de los servicios de vino & 3.64 & 2.7 & -0.94
\end{tabular}

Fuente: Elaboración propia 
Una vez obtenida la medición de la importancia y el grado de presencia percibida así como la brecha existente entre el estado actual y el deseado de cada beneficio buscado, se cuenta con elementos suficientes para definir en esta etapa las oportunidades de mejora de los mismos en dependencia de la posición actual ocupada, lo cual podría constituir un punto de apoyo importante para la toma de decisiones relacionadas con la planificación y ejecución de acciones de mejora que permitan mantener o mejorar el estado actual al nivel deseado.

En la matriz se pudo observar que los beneficios que se ubican en el cuadrante de Alta importancia - Alto grado de presencia percibido son: calidad del servicio, habilidades mostradas por el personal, ambientación/atmósfera del lugar, climatización. A pesar de que en este cuadrante se encuentran las principales fortalezas del servicio, es necesario resaltar que todos estos atributos o beneficios tienen una puntuación por debajo de " 4 " puntos y en todos los casos también se perciben por debajo de la importancia otorgada por lo que constituyen la máxima prioridad a mejorar en el servicio de Alimentos y Bebidas.

En el cuadrante Alta importancia - Bajo grado de presencia percibida se encuentran los beneficios: relación calidad/precio, variedad, tipo de cocina/especialidad. Aquí se identifican las debilidades que presenta el servicio, y a las que se debe prestar una atención especial. Teniendo en cuenta los resultados obtenidos en el análisis del primer cuadrante constituyen estos atributos la segunda prioridad.

En el cuadrante Baja importancia - Bajo grado de presencia percibida se encuentran los atributos: cualidades físicas de las instalaciones, comunicación y publicidad del producto y complementariedad de los servicios de vino. Aquí se ubican los atributos valorados con un bajo grado de presencia percibida y tienen baja prioridad para el cliente.

En el cuadrante Baja importancia- Alto grado de presencia percibida se encuentran los atributos: tipo de servicio, horario de servicio. En estos beneficios el servicio tiene una superioridad irrelevante, ya que el cliente les concede escasa importancia.

Como resultado del análisis mediante la Matriz Importancia-Grado de presencia percibida empleada en el procedimiento, se pudo visualizar cuáles constituyen puntos fuertes del servicio desde la perspectiva de los clientes que lo consumen, así como también los puntos críticos, como se muestra en la tabla 4.

También se visualiza la brecha u oportunidad de mejora para cada beneficio buscado, lo que constituye un punto de apoyo importante para el Hotel Playa Caleta de Varadero en la toma de decisiones relacionadas con la prestación de sus servicios en el proceso de Alimentos y Bebidas.

Los beneficios que representan puntos fuertes para los servicios del proceso, según el análisis anteriormente realizado, presentan una brecha negativa, demostrando que a pesar de ser puntos fuertes en el servicio, constituyen oportunidades de mejora para el mismo, con el objetivo de poder llegar a cumplir con las expectativas de los clientes, potenciando aquellos beneficios que mejor percepción tiene con relación al resto de ellos.

En el caso de los beneficios que constituyen debilidades en el proceso, hay que prestarles una especial atención ya que representan aquellos atributos peor valorados por el cliente y son los que más influencia negativa le transfieren al servicio brindado por el hotel.De acuerdo con el análisis efectuado las acciones de mejora para aumentar la presencia percibida de estos beneficios en el proceso deben centrarse en los beneficios que se ubican en los cuadrantes alta importancia - alto grado de presencia percibida (Puntos fuertes del proceso) y alta importancia - bajo grado de presencia percibida (Puntos críticos), así como en la brecha existente en cada uno de ellos. 
Tabla 4.

Beneficios o atributos que constituyen puntos fuertes y débiles del servicio de restauración.

\begin{tabular}{lccc}
\hline Puntos fuertes & Brecha & Puntos débiles & Brecha \\
\hline Calidad del servicio & -1.63 & Relación calidad/precio & -1.9 \\
Habilidades mostradas por el personal & -1.17 & Variedad, tipo de cocina/ & -1.98 \\
Ambientación/atmósfera del lugar & -0.84 & & \\
Climatización & -0.36 & & \\
\end{tabular}

Fuente: Elaboración propia

El programa de mejora debe estar en función de aquellos beneficios que son más importantes para los clientes, y que obtengan un grado de presencia percibida adecuado con el objetivo de mantener sus principales fortalezas competitivas; entre ellos se concentran: los beneficios tales como calidad del servicio, habilidades mostradas por el personal, ambientación/atmósfera del lugar y climatización.

Cabe mencionar que aunque estos beneficios constituyen las fortalezas del proceso, debe procurarse la máxima prioridad de mejora hacia ellos, debido que en su totalidad presentan una brecha negativa entre ambos estados. Los puntos críticos o débiles del proceso (cuadrante alta importancia-bajo grado de presencia percibida) deben ser gestionados de igual forma, pues representan el nivel insatisfacción de los clientes con un bajo grado de presencia percibida en los beneficios buscados por ellos, que en orden de prioridad están enfocados: relación calidad/ precio y variedad, tipo de cocina/especialidad.

La segunda etapa del procedimiento es determinar el Nivel de Servicio ofertado con la aplicación del método propuesto por Conejero et.al. (2008) a partir de la multiplicación del peso o grado de importancia de los atributos y su evaluación por parte del cliente.

Para determinar el peso o grado de importancia de los atributos se puede aplicar los métodos de comparación pareada como el Triángulo de Füller, el Expert Choice, entre otros.

Para ello fue necesario determinar en primer lugar el peso de cada criterio según los expertos, aplicando el Triángulo de Füller Modificado.

Para llevar a cabo esta comparación por pares, se elabora una matriz criterio-criterio como se muestra en el Tabla 5, donde se consigan las preferencias en cada pareja de criterios (1 significa que el criterio i es más importante que el criterio j). Cada $\mathrm{Ci}$ y $\mathrm{Cj}$ corresponde a los atributos del servicio determinados a través del método Delphi y que ya fueron explicados anteriormente. 
Tabla 5.

Matriz Criterio-Criterio.

\begin{tabular}{|c|c|c|c|c|c|c|c|c|c|c|c|}
\hline & $\mathrm{C1}$ & C 2 & $C 3$ & C4 & C5 & C6 & C 7 & C8 & C9 & C 10 & C 11 \\
\hline C1 & 1 & 1 & 0 & 0 & 0 & 0 & 1 & 0 & 0 & 0 & 0 \\
\hline C2 & 0 & 1 & 0 & 0 & 0 & 0 & 1 & 0 & 0 & 0 & 0 \\
\hline C3 & 1 & 1 & 1 & 1 & 1 & 1 & 1 & 0 & 0 & 1 & 1 \\
\hline $\mathrm{C4}$ & 1 & 1 & 0 & 1 & 1 & 1 & 1 & 0 & 0 & 0 & 1 \\
\hline C5 & 1 & 1 & 0 & 0 & 1 & 1 & 1 & 0 & 0 & 0 & 0 \\
\hline C6 & 1 & 1 & 0 & 0 & 0 & 1 & 1 & 0 & 0 & 1 & 0 \\
\hline C7 & 0 & 0 & 0 & 0 & 0 & 0 & 1 & 0 & 0 & 0 & 0 \\
\hline C8 & 1 & 1 & 1 & 1 & 1 & 1 & 1 & 1 & 1 & 1 & 1 \\
\hline $\mathrm{Cg}$ & 1 & 1 & 1 & 1 & 1 & 1 & 1 & 0 & 1 & 1 & 1 \\
\hline $\mathrm{C}_{10}$ & 1 & 1 & 0 & 1 & 1 & 0 & 1 & 0 & 0 & 1 & 0 \\
\hline C11 & 1 & 1 & 0 & 0 & 1 & 1 & 1 & 0 & 0 & 1 & 1 \\
\hline
\end{tabular}

Fuente: Elaboración propia

En la tabla 6 se muestran los valores respectivos, y el peso subjetivo resultante de cada criterio, siendo:

$\mathrm{W}=\Sigma$ de selecciones de cada criterio/Total de comparaciones de los criterios

Tabla 6.

Pesos subjetivos de los criterios.

\begin{tabular}{lccc}
\hline Criterios & $\sum$ & W & Peso (\%) \\
\hline 1-Cualidades físicas de las instalaciones & 3 & 0.05 & 5 \\
2-Comunicación y publicidad del producto & 2 & 0.03 & 3 \\
3-Variedad, tipo de cocina/especialidad & 9 & 0.14 & 14 \\
4-Tipo de servicio & 7 & 0.11 & 11 \\
5-Ambientación/Atmósfera del lugar & 5 & 0.08 & 8 \\
6-Climatización & 5 & 0.08 & 8 \\
7-Horario de servicio & 1 & 0.02 & 2 \\
8-Relación calidad/precio & 11 & 0.17 & 17 \\
9-Calidad del servicio & 10 & 0.15 & 15 \\
10-Habilidades mostradas por el personal & 6 & 0.09 & 9 \\
11-Complementariedad de los servicios de vino & 7 & 0.11 & 11 \\
\hline
\end{tabular}

Fuente: Elaboración propia 
Después de hallar el peso de cada criterio se concluyó que el orden de importancia es el siguiente:

1. Relación calidad/precio

2. Calidad del servicio

3. Variedad, tipo de cocina/especialidad

4. Tipo de servicio

5. Complementariedad de los servicios de vino

6. Habilidades demostradas por el personal.

7. Ambientación/atmósfera del lugar

8. Climatización

9. Cualidades físicas de las instalaciones

10. Comunicación y publicidad del producto

11. Horario de servicio

Una vez determinado el peso se calcula la frecuencia de cada uno de los criterios mediante el software SPSS versión 20.0 para determinar la puntuación o evaluación del atributo, como se muestra en la tabla 7.

Tabla 7.

Puntuaciones obtenidas por cada atributo.

\begin{tabular}{lc}
\hline Atributo & Puntuación \\
\hline Cualidades físicas de las instalaciones & 3 \\
Comunicación y publicidad del producto & 2 \\
Variedad, tipo de cocina/especialidad & 2 \\
Tipo de servicio & 3 \\
Ambientación/Atmósfera del lugar & 4 \\
Climatización & 5 \\
Horario de servicio & 4 \\
Relación calidad/precio & 4 \\
Calidad del servicio & 9 \\
Habilidades mostradas por el personal & 3 \\
Complementariedad de los servicios de vino & 4 \\
\hline
\end{tabular}

Fuente: Elaboración propia

Una vez conocidos los valores necesarios, se procede al cálculo del Nivel de Servicio, obteniéndose un nivel de servicio ofertado del $70 \%$ que al compararse con los estándares establecidos por la cadena (92.25\%) para un Hotel 4 estrellas, queda en un $22.25 \%$ por debajo.

Los atributos que más afectan el resultado alcanzado y en los que se debe trabajar para su disminución son:

1. Comunicación y publicidad del producto

2. Horario de servicio

3. Cualidades físicas de las instalaciones

4. Variedad, tipo de cocina/especialidad

5. Habilidades mostradas por el personal

6. Ambientación/Atmósfera del lugar 
7. Tipo de servicio

8. Climatización

9. Complementariedad de los servicios de vino

10. Calidad del servicio

11. Relación calidad/precio

Para lograr una mejora de los procesos de Alimentos y Bebidas, de acuerdo con el análisis efectuado, las acciones de mejora para aumentar la presencia percibida de los atributos en el proceso deben centrarse en los beneficios que se ubican en los cuadrantes Alta importancia Alto grado de presencia percibida (Puntos fuertes del proceso) y Alta importancia - Bajo grado de presencia percibida (Puntos críticos) de la matriz analizada anteriormente, así como en la brecha existente en cada uno de ellos, las que reflejan cuán lejos está el cumplimiento de los deseos del cliente.

El programa de mejora debe estar en función de aquellos beneficios que son más importantes para los clientes, y que obtengan un grado de presencia percibida adecuado con el objetivo de mantener sus principales fortalezas competitivas; entre ellos se concentran: los beneficios tales como calidad del servicio, habilidades mostradas por el personal, ambientación/atmósfera del lugar y climatización.

Aunque estos beneficios constituyen las fortalezas del proceso, debe procurarse la máxima prioridad de mejora hacia ellos, debido que en su totalidad presentan una brecha negativa entre ambos estados. Los puntos críticos o débiles del proceso deben ser gestionados de igual forma, pues representan el nivel insatisfacción de los clientes con un bajo grado de presencia percibida en los beneficios buscados por ellos, que en orden de prioridad están enfocados: relación calidad/precio y variedad, tipo de cocina/especialidad conteniendo una brecha de -1.9 y -1.98 respectivamente. Partiendo del diagnóstico y análisis de situación la entidad debe proponer un plan de mejoras que responda a los problemas encontrados.

\section{Discusión}

Para el diseño correcto de un proceso servuctivo es necesario realizar previamente un estudio de los atributos que deben estar presentes en el servicio que se brinda, los cuales son evaluados por los clientes, de cuyo análisis es posible obtener las brechas o desviaciones en relación a la presencia percibida por estos y que deben ser incorporadas al plan de mejora de dicho proceso.

Es pertinente aplicar la fórmula del nuevo servicio en la restauración, la cual comprende cinco decisiones claves que se interesan por el concepto, el segmento, la servucción, la oferta y la imagen, todo lo cual incide de manera directa en el nivel de servicio ofertado.

\section{Conclusiones}

Existe una creciente base teórica-conceptual sobre la gestión de servicios de restauración, sin embargo, quedan espacios para la investigación teórica y práctica en relación a la aplicación del enfoque servuctivo como vía para lograr un mejor desempeño de las entidades que lo prestan.

Los estudios precedentes consultados sustentaron la pertinencia de la investigación realizada y la necesidad de diseñar un procedimiento metodológico para el estudio de procesos de restauración, en el que se integran diferentes enfoques de gestión de los servicios como calidad, servucción, marketing, procesos, siendo posible la implementación del nuevo concepto de servicio. 
A partir del trabajo con expertos y la aplicación del método Delphi se pudieron determinar los 11 atributos que caracterizan el proceso de servicio de restauración, a través de los cuales fue factible determinar el nivel de presencia de estos y su incidencia en la calidad percibida por los clientes.

Se pudo determinar que el Nivel de Servicio ofertado tiene un valor del 70\%, siendo inferior en un $22,25 \%$ con respecto al estándar de la cadena para un hotel 4 estrellas, evidenciándose las insuficiencias detectadas.

El análisis de los resultados de la valoración por los clientes del nivel de importancia y el grado de presencia percibida de los atributos del servicio de restauración que han consumido, fue complementado con la aplicación de la Matriz Importancia-Grado de Presencia Percibida, lo que permitió establecer las prioridades para la mejora en aquellos atributos que sean más importantes para los clientes y que estén menos adecuados.

\section{Referencias}

Aboal et.al. (2009). La innovación y la nueva economía de servicios en América Latina y el Caribe Retos e implicaciones de política. Editorial Manosanta Desarrollo. Centro de Investigaciones Económicas CINVE, Uruguay.

Amozarrain (1999) La gestión por procesos. Editorial Mondragón Corporación Cooperativa. España.

Betancourt-Ramírez, José Bernardo; Aldana-de-Vega, Luzangela; Gómez-Betancourt, Gonzalo (2014). Servicio, ambiente y calidad de restaurantes en Bogotá. Estudio comparativo de empresa familiar y empresa no familiar. Revista Entramado, 10(2), 60-74. ISSN: 1900-3803. Universidad Libre. Colombia.

Briceño de Gómez y García de Berrios (2008). La servucción y la calidad en la fabricación del servicio. Revista Visión Gerencial. 7(1),21-32. ISSN: 2477-9547. Universidad de los Andes. Venezuela.

Cisneros Mustelier y Hechavarría Veira (2011) Factores que inciden en el costo de un servicio de bufet en un hotel citadino. Revista Contaduría y Administración. 233, 55-72. ISSN 0186-1042. Universidad Nacional Autónoma de México.

Diéguez Matellán, E. (2008). Contribución a la planificación de servicios complementarios extrahoteleros en destinos turísticos. Aplicación Varadero. Tesis presentada en opción al grado científico de Doctor en Ciencias Técnicas. Universidad de Matanzas. Cuba.

Denton, K (1991). Calidad en el servicio a los clientes. Ediciones Díaz de Santos S.A. España.

Duque Oliva, E. (2005). Revisión del concepto de calidad del servicio y sus modelos de medición. Innovar, Revista de Ciencias Administrativas y Sociales 15 (25), 64-80. ISSN: 0121-5051. Universidad Nacional de Colombia.

Eigler, P y Langeard, E (1989). Servucción, el marketing de servicios. McGraw-Hill / Interamericana de España.

Espinosa, J. M. (2010) Gestión de la Restauración. Editorial pueblo y educación, Ciudad de La Habana, Cuba.

George, D., y Mallery, P. (2003). SPSS for Windows step by step: A simple guide and reference. 11.0 update (4 ed.). Boston, MA: Allyn \& Bacon.

Grönroos, C. (1994). Marketing y gestión de servicios: la gestión de los momentos de la verdad y la competencia en los servicios. Madrid: Editorial Díaz de Santos.

Grönroos, C. (2001). The perceived service quality concept a mistake? Managing Service Quality. 11(3), $150-152$.

Harrington, H.J. (1998). Mejoramiento de los procesos de la empresa. Mc Graw-Hill, Santa Fe de Bogotá, Colombia.

Hernández González, Y. (2008) Propuesta metodológica y aplicación de un procedimiento de diseño de sistemas servuctivos en el área de Recepción del Hotel Arenas Doradas. Tesis presentada en opción al título de Ingeniería Industrial. Universidad de Matanzas. Cuba.

Hernández Torre, M. (2008). Diagnóstico para la gestión del proceso de cambio integrado. Revista Ingeniería Industrial. 29(1),3-7. ISSN: 1815-5936

Mantilla, J. A. \& James, L. C. (1977) “Importante-Performance Análisis”. Journal of Marketing. 41 (1), 13-17. 
Medina León, A., D. Nogueira Rivera, et al. (2012). Consideraciones y criterios para la selección de procesos para la mejora: Procesos Diana. La Habana, Revista de Ingeniería Industrial. 33 (3), 272-281. ISSN: 1815-5936

Morillo Moreno et.al. (2011). Medición de la calidad del servicio en las instituciones financieras a través de la escala del SERVQUAL. Contaduría y Administración. (234), 101-130. ISSN 0186-1042. Universidad Nacional Autónoma de México.

Negrín Sosa (2003). El Mejoramiento de la Administración de operaciones en Empresas de servicios hoteleros. Tesis presentada en opción al grado científico de Doctor en Ciencias técnicas. Universidad de Matanzas. Cuba.

Nogueira Rivera (2002). Modelo Conceptual y herramientas de apoyo para potenciar el Control de gestión en las empresas cubanas. Tesis presentada en opción al grado científico de Doctor en Ciencias técnicas. ISPJAE. Cuba.

OCDE (Organizacion para la Cooperacion y el Desarrollo Economicos) (2010). Science, Technology and Industry Outlook 2010. Paris: OCDE.

Parra Ferié, C. (2005). Modelo y procedimientos para la gestión con óptica de Servucción de los servicios técnicos automotrices como elemento del sistema turístico cubano. Tesis en opción al grado científico de Doctor en Ciencias técnicas. Universidad de Matanzas. Cuba.

Parra Ferié, C. (2009). Procesos de servicios. Tendencias modernas en su gestión. Editorial Universitaria MES.

Pride y Ferrell (1997). Marketing. Conceptos y estrategias. 9na edición. Ediciones McGraw-Hill. México.

Stefano, Casarotto Filho \& Godoy (2010). Medición de la calidad percibida en organización de servicio: utilizando o Índice de Potencial de Aumento de la Satisfacción del Consumidor (PASC) y Modelo dos 5 GAPS. Revista Electrónica Sistemas \& Gestión, 5 (19, 17-31. ISSN, 2410-3977. 